\title{
An Intraoral Repair Method for Chipping Fracture of a Multi-unit Fixed Zirconia Reconstruction: A Direct Dental Technique
}

\author{
Alfredo Mikail Melo Mesquita ${ }^{1}$ Nadin Al-Haj Husain² \\ 1Department of Prosthodontics, Paulista University (UNIP), \\ São Paulo, Brazil \\ 2Department of Reconstructive Dentistry and Gerodontology, \\ School of Dental Medicine, University of Bern, Bern, Switzerland \\ ${ }^{3}$ Division of Dental Biomaterials, Center for Dental and Oral Medicine, \\ Clinic for Reconstructive Dentstry, University of Zurich, Switzerland
}

Pedro Molinero-Mourelle² Mutlu Özcan ${ }^{3}$

\author{
Address for correspondence Mutlu Özcan, DDS, DMD, PhD, \\ Division of Dental Biomaterials, Center for Dental and Oral \\ Medicine, Clinic for Reconstructive Dentstry, University of Zurich, \\ Plattenstrasse 11, CH-8032 Zurich, Switzerland \\ (e-mail: mutluozcan@hotmail.com)
}

Eur J Dent:2021;5:174-178

\begin{abstract}
Keywords

- ceramic

- chipping

- reveneering

- zirconia

Fracture or chipping of veneering ceramic is one of the most frequent clinical failures in dentistry in fixed dental and implant-borne prostheses. Due to the friable nature of the ceramic material, chippings may result in an aesthetic and functional problem for the patient requiring a rapid solution. Direct repairs have been indicated for the restoration of function, aesthetics and comfort, especially in cases where the fractured prosthesis presents good adaptation and satisfactory aesthetics. This case report aims to present and discuss the techniques of the direct reveneering method, their advantages and disadvantages, as well as the importance of adhesive procedures in the success of these restorative approaches. Success in repairing the fractured area, regardless of the technique used, is fundamental to establish a strong and stable adhesion between the repaired and the fractured areas, since the longevity of the repair will depend on the quality of the interface generated.
\end{abstract}

\section{Introduction}

In the past decades, socioeconomic changes and improvements of worldwide oral health systems have caused a decrease in the edentulous and an increase in the partially edentulous humans. These changes allowed an expansion of the treatment options for missing teeth..$^{1-3}$ Fixed dental prostheses (FDPs) are a treatment option for the replacement of one or more missing teeth in partially edentulous patients. Although the gold standard in FDPs is still ceramic fused to metal, this material can present aesthetic shortcomings and requires a more invasive tooth preparation. ${ }^{4-7}$

FDPs based on full-ceramic systems have gained importance due to their good aesthetical and biological results fulfilling increasing aesthetical demands of patients and clinicians. ${ }^{78}$ This demands promoted the development of several ceramic systems for FDPs, in which the yttria-stabilized tetragonal zirconia polycrystal is one of the most

DOI https://doi.org/ 10.1055/s-0040-1716311 ISSN 1305-7456. used materials due to its good aesthetic, biological, and mechanical properties.9.10 However, these restorations are also prone to clinical complications. Chipping of the veneering ceramic is the most common complication, followed by the framework fractures. ${ }^{11}$

The clinical performance of double-layered zirconia FDPs has similar chipping rates compared with metal ceramic. Due to its excellent mechanical properties, successfully achieving structural demands, zirconia is considered as a favorable material choice. ${ }^{12}$ Nevertheless, the bonding of the low resistance coating ceramic to the zirconia core can present tension zones at the interfaces, which can generate microcracks, resulting in chippings or fractures. ${ }^{13}$ Such failures are caused by thermal stress after sintering or lack of surface preparation for the coating. Furthermore, these technical complications may also be associated with clinical and laboratory risk factors such as inadequate infrastructure design, dental preparation, lack of ceramic polishing, and parafunctional habits. ${ }^{12,13}$

(C) 2020. European Journal of Dentistry.

This is an open access article published by Thieme under the terms of the Creative Commons Attribution-NonDerivative-NonCommercial-License, permitting copying and reproduction so long as the original work is given appropriate credit. Contents may not be used for commercial purposes, or adapted, remixed, transformed or built upon. (https://creativecommons.org/licenses/by-nc-nd/4.0/)

Thieme Medical and Scientific Publishers Pvt. Ltd., A-12, 2nd Floor, Sector 2, Noida-201301 UP, India 
Chippings or framework fractures can have a negative impact on the quality of life of patients because of impairment in aesthetics and function, as well as the caused cost and time loss of additional repair. These complications are especially problematic in cases of multiunit restorations containing more than three units. ${ }^{1214}$ The repair process includes the removal, fabrication, and placement of a provisional restoration or subsequent repair and/or rebonding. To avoid complete replacement in medically or prosthetically challenging situation, clinicians have the possibility of a repair procedure based on direct and chairside repair techniques. ${ }^{13-15}$ However, the current evidence of their success is limited. Furthermore, the combination of increased variability of materials and fabrication technologies necessitates the development of suitable clinical repair protocols.

The aim of this dental technique was therefore to describe a repair protocol for ceramic-zirconia restorations for cases of multiunit tooth-supported FDP chipping fracture.

\section{Dental Technique}

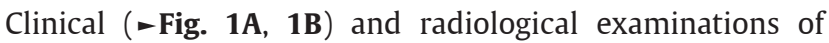
the fractured area were conducted. The fracture of zirconia bridge with the infrastructure (Lava; 3M ESPE AG, Seefeld, Germany) and veneering ceramic (IPS e.max Ceram; Ivoclar Vivadent, Schaan, Liechtenstein) occurred 11 months after cementation. The cementation was performed using the self-adhesive resin cement (RelyX U200, 3M ESPE AG), after sandblasting (30 $\mu \mathrm{m} \mathrm{SiOx}$, CoJet-Sand, 3M ESPE AG) and silane application (Monobond, Ivoclar Vivadent). Possible premature contacts were examined throughout occlusion control using occlusion paper and eliminated. ${ }^{16}$ The future shade of the repair composite was determined, and the veneering and core ceramic were cleaned using fluoride-free paste. The glaze of the veneering ceramic was thereafter removed from the margins of the repair using a fine-grit diamond rotatory bur with a shoulder edge (FG 861; Intensiv, Montagnola, Switzerland) under water cooling, creating a bevel shape at the future bonding area. ${ }^{17}$ Afterward, glycerin gel was applied on the complete area except the beveled one, to create a coating layer. Thereafter, the area has been isolated using a rubber dam. The zirconia surface was air

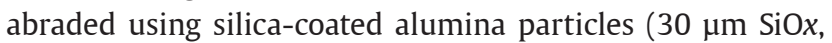
CoJet-Sand, 3M ESPE AG) through an intraoral air abrasion

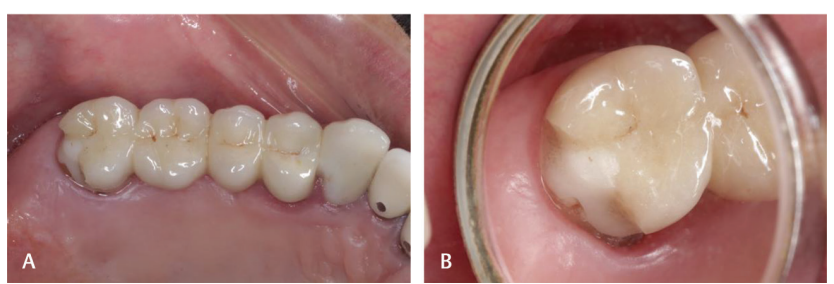

Fig. 1 (A) Patient presents all-ceramic veneering and core fracture at the distal and occlusal area of veneered zirconia fused to multiunit zirconia bridge at tooth 17 , (B) Closeup view of veneering and core fracture of 17 . device (Korox, Bego, Bremen, Germany) at a pressure of 2.5 bar from a distance of $\sim 10 \mathrm{~mm}$ for 5 seconds ( - Fig. 2 ). Afterward, the zirconia veneering and thereafter core surface were rinsed ( - Fig. 3A, 3B), air dried, and etched using hydrofluoric (HF) acid (Ultra-Etch; Ultradent, South Jordan, Utah, United States) for 90 seconds and rinsed for 90 seconds and air dried. A 3-methacryloxypropyltrimethoxy silane coupling agent (ESPE-Sil, 3 M ESPE AG) was applied to the surface of the restoration for 60 seconds and air dried. Thereafter, the adhesive resin (StickResin; StickTech, Turku, Finland) was applied to the entire surface to be bonded ( - Fig. 4 ) and photopolymerized (Optilux 501, Kerr, West Collins, Orange, California, United States) for 20 seconds ( - Fig. 5). The resin composite (Clearfil Photoposterior, Kuraray, Japan) was applied and bonded to the conditioned surfaces incrementally and photopolymerized for 40 seconds ( - Fig. 6). The occlusion was checked using articulating paper (Hanel Articulating Paper, Coltène/Whaledent, Inc, Switzerland). Premature contacts were eliminated and finally, the restoration was refinished and repolished ( $\mathbf{- F i g . ~ 7 A , 7 B}$ ). The finishing and polishing procedures were performed using the polishing discs set (Astropol, Ivoclar Vivadent).

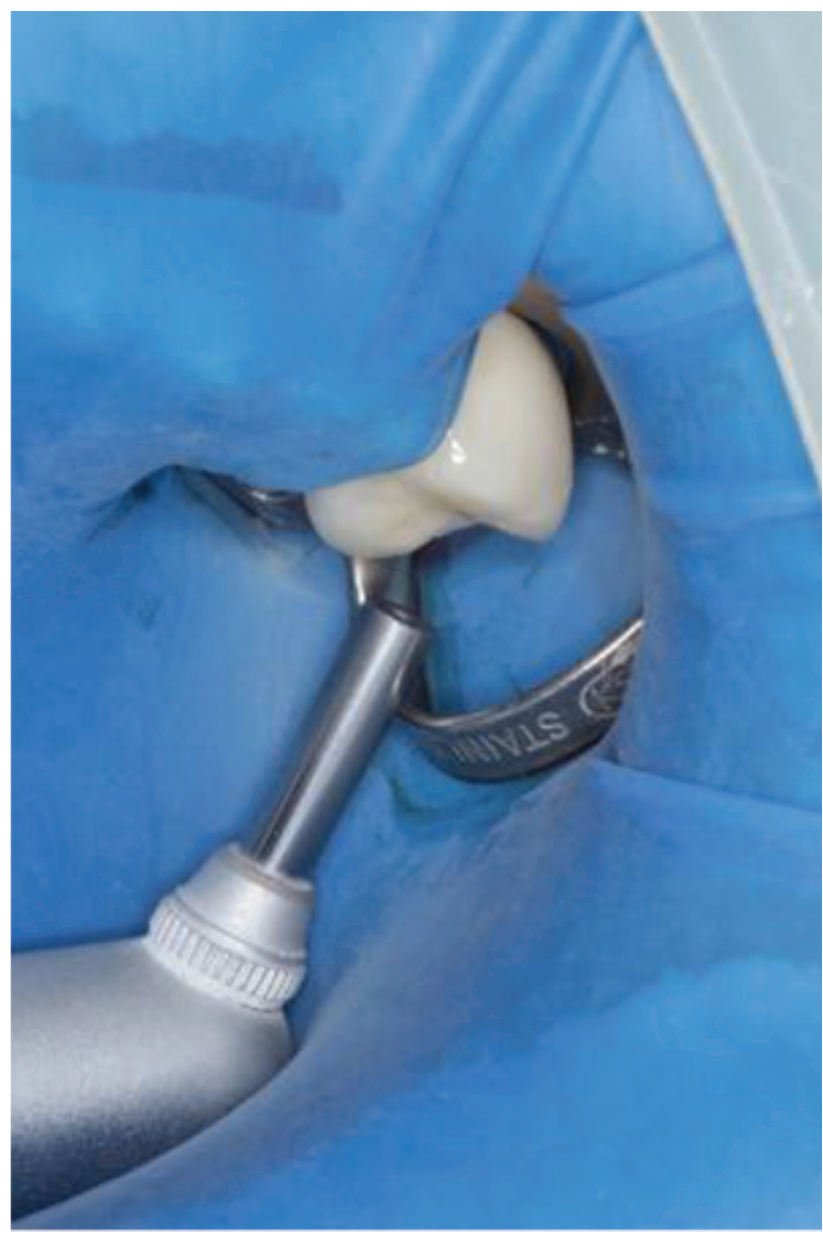

Fig. 2 Air abrasion using a chairside device with silica-coated alumina particles ( $30 \mu \mathrm{m}, 2.5$ bar for 20 seconds). 

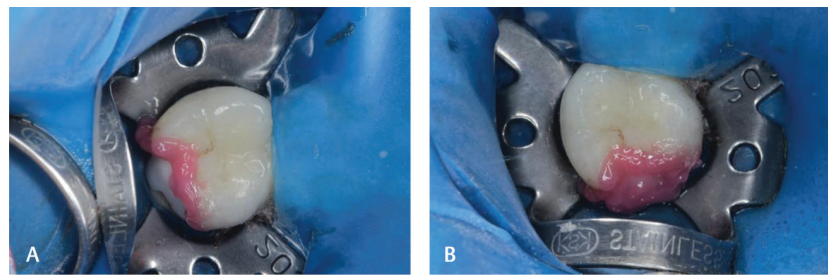

Fig. 3 (A, B) Etching of the fractured area with 5\% hydrofluoric acid for 20 seconds of the $(\mathbf{A})$ veneering and $(\mathbf{B})$ core ceramic.

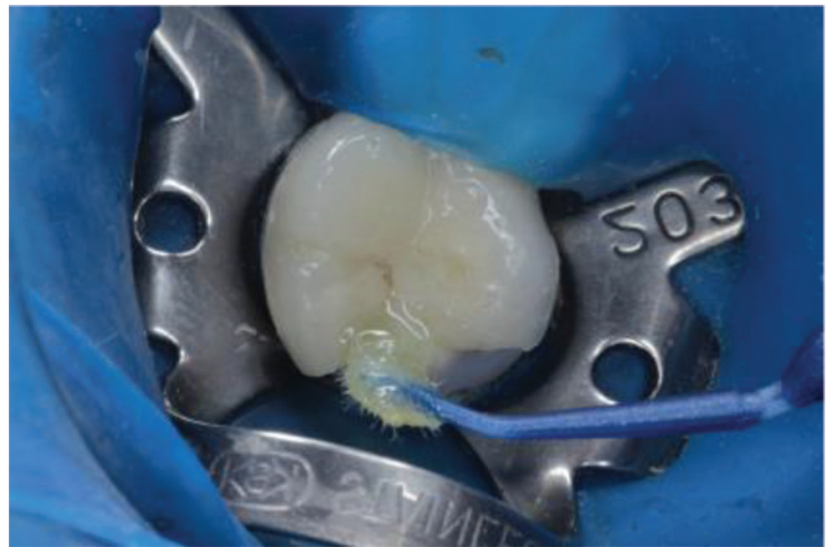

Fig. 4 Application of adhesive resin.

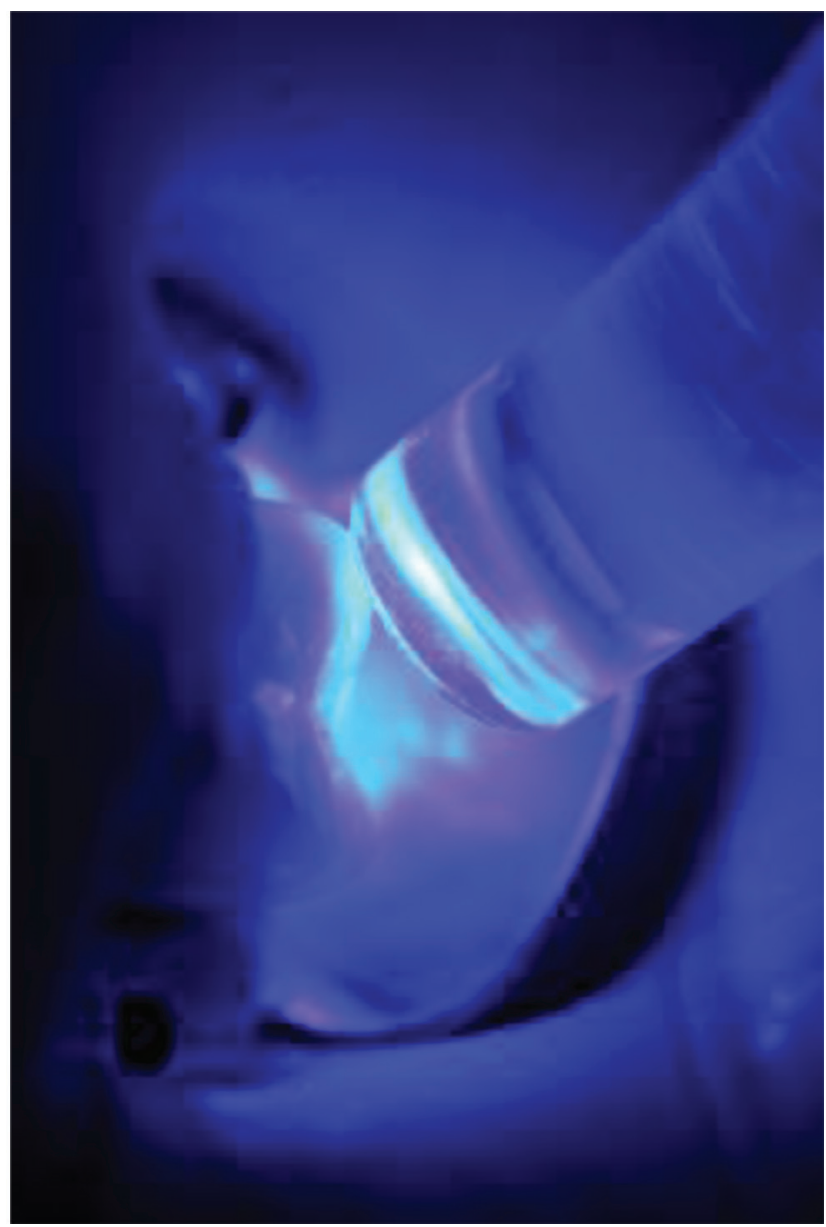

Fig. 5 Photopolymerization of the adhesive resin.

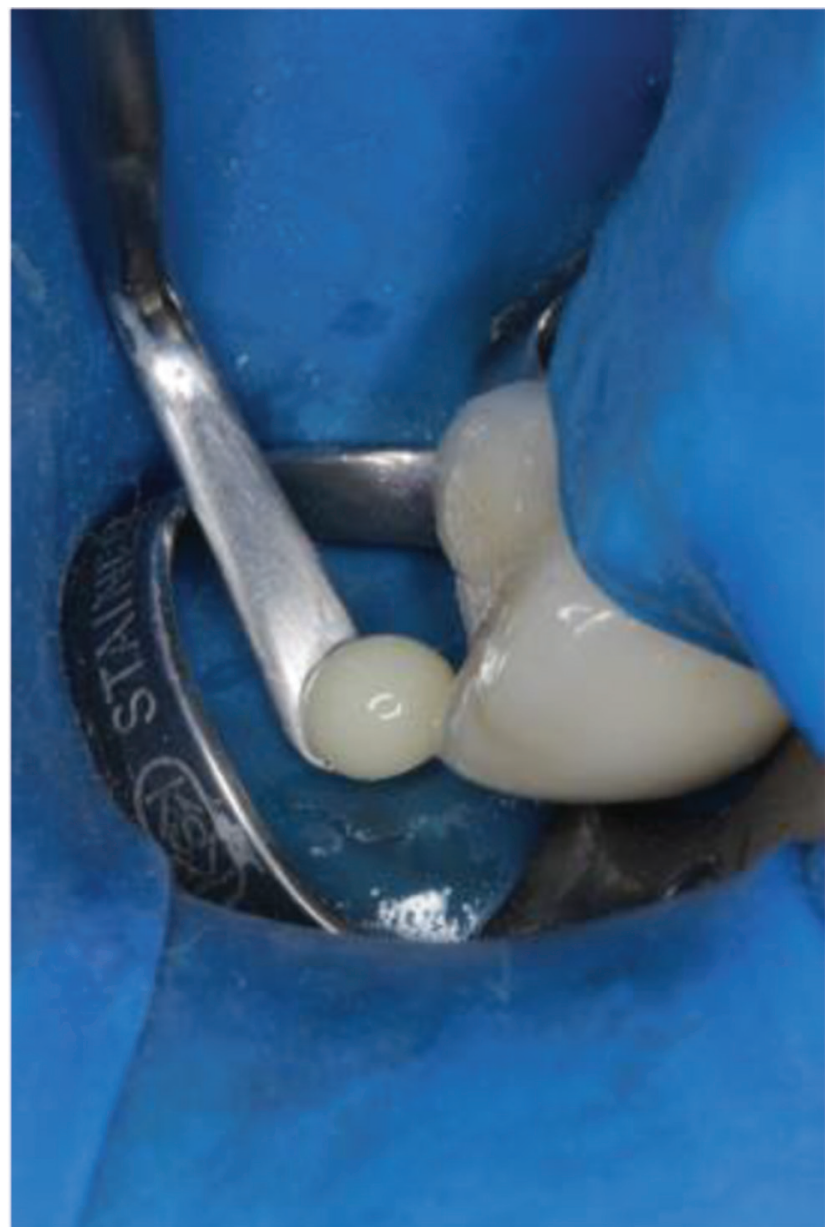

Fig. 6 Application of resin composite incrementally.

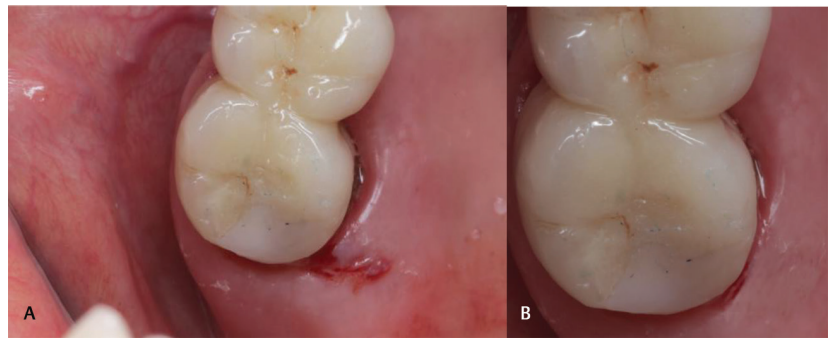

Fig. 7 Final (A) over and (B) closeup view of repaired distal palatinal veneering and core fracture.

\section{Discussion}

When considering the need to repair a chipping or core fracture directly, the size and location of the failure must be considered carefully, especially in multiunit restorations. Moreover, the prosthesis should be evaluated clinically and radiographically, as such complications might cause aesthetic problems and discomfort due to sharp edges. The initial clinical evaluation is essential to carry out a correct treatment plan. In cases where the FDPs present a good marginal fit and a satisfactory aesthetic, it is possible to choose the direct reveneering option. However, if future complications/failures are not tolerated by the patient, the fabrication of a new prosthesis is preferable. ${ }^{13,15}$ During the 
clinical evaluation procedure, the type of fracture will be evaluated, as it determines the future adhesive protocol to be used during the repair. Two types of failure can occur, the chipping with exposure of the infrastructure or ceramic veneering chipping without the exposure of the core. ${ }^{13,14}$

Very small failures are considered favorable since they can be easily resolved throughout polishing with rubber tips. Small and medium failures are resolved using direct repairs with composite resin, ${ }^{15}$ while large chippings including interproximal and occlusal areas require indirect repair protocols in the dental laboratory or are even considered for refabrication. ${ }^{15,18}$

The objective of the repair is based on achieving a strong and stable adhesion between the repair and the fractured areas. Its longevity depends on the quality of the interface created during the repair. The use of resin composite allows the failure to be corrected quickly, effectively, and economic favorably. This approach can be done chairside in a single session with few additional costs. ${ }^{15}$

The composite resin is a material of easy clinical handling, which fits perfectly in the area to be restored, and presents a longer working time. For the patient, the rapid resolution of the aesthetic and functional problem generates confidence in the treatment, especially if the prosthetic restoration in question is recent and is located in the anterior region. However, the color of the intraoral repair will not always be the same as the existing color, and this is due to the difference between the materials used (composite resin and ceramic). In addition, the wear of the composite resin is much greater than that of the ceramic and may lead to the need for more frequent replacements. ${ }^{8}$ These situations must be informed to the patient at the moment of decision making, since they are important limitations of this technique. ${ }^{16}$

In cases of veneering fracture and especially core exposure, surface treatment should be performed, which is usually based on sandblasting with or without silica deposition, primers, and bonding agents.

The occlusion checking avoids possible problems and the color selection must be done beforehand. The use of insolation using rubber dam should be done whenever possible to maintain a clean pellicle and contaminant-free surface. ${ }^{14}$

Removal of the glaze of the veneering ceramic using rotating diamond burs aims to create a bevel shape resulting in a smooth transition between the ceramic and composite resin surfaces by allowing a better reaction of the silane coupling agent with the ceramics. ${ }^{14}$

Air abrasion procedure should only be performed on the glaze-free surface, as it may harm the remaining ceramic. Therefore, the uninvolved areas should be protected using glycerine gel or a polyfluoroethylene tape. In case of metal or zirconia exposure, air abrasion using a chairside air particles coated with silica or silica only (particle size range: $30-50 \mu \mathrm{m}$, blasting pressure: 2.5 bar) for $\sim 5$ seconds in circling motion at a distance of $\sim 10 \mathrm{~mm}$ should be performed. ${ }^{14}$ On vitreous surfaces, $70 \%$ alcohol is used for an initial cleaning, and soon after, acid conditioning is done with $10 \% \mathrm{HF}$ acid for 2 to 3 minutes (feldspar porcelain), 1 minute (leucite), or
20 seconds (disilicate). Ceramic margins that have been worn are conditioned with $\mathrm{HF}$ acid $9.6 \%$ for 90 seconds. ${ }^{14}$

On the clean and dry surface, the silane coupling agent is applied with a disposable brush for 1 minute and the surface is dry with air jets. Metal and zirconia surfaces should be masked with an opaque resin. The resin should be taken with the tip of an exploratory probe over the metal/zirconia, making sure that it is not placed on the edges of the bevel. Adhesive agent is applied to the ceramic surfaces, refined by aspiration and photopolymerized for 20 seconds according to the manufacturer's recommendations. ${ }^{16}$

Incremental application of composite resin helps control the polymerization shrinkage, increasing the longevity of intraoral repair.

As for indirect repairs, in the case of more extensive fractures and with exposed cores, more complex procedures are required, with possible invasive preparations and mechanical and chemical surface treatments. ${ }^{19-21}$ In addition, dental impressions are needed to manufacture the new fragment in the laboratory. However, this technique is more durable and presents better color stability, yet time consuming and expensive. ${ }^{14-16}$ It is important to inform the patient that the removal of the restoration can cause clinical complications such as fractures or dental wear.

Throughout choosing a direct repair option, it will not be possible to achieve the same performance presented by an indirect laboratory solution. However, it presents a quick and efficient solution to possible problems arising from the fracture or chipping of the veneering ceramic, without the need for replacement of the prosthesis.

The success of these approaches depends to a great extent on the knowledge and handling of these materials and techniques by the clinician and the accurate and meticulous execution of the instructions and clinical protocolized steps.

\section{Clinical Significance}

When direct repair techniques are well indicated and performed, they help solve, quickly and efficiently, the problems arising from the fracture or chipping of the veneering ceramic, without the need for replacement of the prosthesis. However, success in repairing the fractured area is fundamental to establish a strong and stable adhesion. The longevity of the repair will depend on the quality of the interface generated as well as on the knowledge and control of the adhesive techniques of the clinician.

\section{Conflict of Interest}

None declared.

\section{References}

1 Abt E, Carr AB, Worthington HV. Interventions for replacing missing teeth: partially absent dentition. Cochrane Database Syst Rev 2012;2(2):CD003814

2 Chan RW, Tseng TN. Single tooth replacement-expanded treatment options. Aust Dent J 1994;39(3):137-149 
3 Salinas TJ, Block MS, Sadan A. Fixed partial denture or single-tooth implant restoration? Statistical considerations for sequencing and treatment. J Oral Maxillofac Surg 2004;62(9, suppl 2) :2-16

4 Anusavice KJ. Standardizing failure, success, and survival decisions in clinical studies of ceramic and metal-ceramic fixed dental prostheses. Dent Mater 2012;28(1):102-111

5 Reitemeier B, Hänsel K, Kastner C, Weber A, Walter MH. A prospective 10 -year study of metal ceramic single crowns and fixed dental prosthesis retainers in private practice settings. J Prosthet Dent 2013;109(3):149-155

6 Näpänkangas R, Raustia A. Twenty-year follow-up of metal-ceramic single crowns: A retrospective study. Int J Prosthodont 2008;21(4):307-311

7 Edelhoff D, Sorensen JA. Tooth structure removal associated with various preparation designs for posterior teeth. Int J Periodontics Restorative Dent 2002;22(3):241-249

8 McLean JW. Evolution of dental ceramics in the twentieth century. J Prosthet Dent 2001;85(1):61-66

9 Manicone PF, Rossi Iommetti P, Raffaelli L. An overview of zirconia ceramics: Basic properties and clinical applications. J Dent 2007;35(11):819-826

10 Zarone F, Russo S, Sorrentino R. From porcelain-fused-tometal to zirconia: clinical and experimental considerations. Dent Mater 2011;27(1):83-96

11 Choi B-K, Han J-S, Yang J-H, Lee JB, Kim SH. Shear bond strength of veneering porcelain to zirconia and metal cores. J Adv Prosthodont 2009;1(3):129-135

12 Limones A, Molinero-Mourelle P, Azevedo L, Romeo-Rubio M, Correia A, Gómez-Polo M. Zirconia-ceramic versus metal-ceramic posterior multiunit tooth-supported fixed dental prostheses: A systematic review and meta-analysis of randomized controlled trials. J Am Dent Assoc 2020;151(4):230-238.e7
13 Özcan M. Fracture reasons in ceramic-fused-to-metal restorations. J Oral Rehabil 2003;30(3):265-269

14 Özcan M. How to repair ceramic chipping or fracture in metal-ceramic fixed dental prostheses intraorally: step-by-step procedures. J Adhes Dent 2014;16(5):491-492

15 Garbelotto LG, Maziero Volpato CA, Rocha Md, Maranghello CA, Calasans A, Ozcan M. Laboratory and clinical considerations on prosthetic zirconia infrastructures for implants. Implant Dent 2013;22(6):578-583

16 Celebi AA, Lee SH, Kau CH. Size discrepancies in molars and first key to optimal occlusion. Eur J Dent 2017;11(2):250-252

17 Ntovas P, Doukoudakis S, Tzoutzas J, Lagouvardos P. Evidence provided for the use of oscillating instruments in restorative dentistry: a systematic review. Eur J Dent 2017;11(2):268-273

18 Agingu C, Zhang CY, Jiang NW, et al. Intraoral repair of chipped or fractured veneered zirconia crowns and fixed dental prosthesis: clinical guidelines based on literature review. J Adhes Sci Technol 2018;32:1711-1723

19 Özcan M, Volpato CA. Surface conditioning protocol for the adhesion of resin-based materials to glassy matrix ceramics: how to condition and why? J Adhes Dent 2015;17(3):292-293

20 Özcan M, Niedermeier W. Clinical study on the reasons for and location of failures of metal-ceramic restorations and survival of repairs. Int J Prosthodont 2002;15(3):299-302

21 Ozer F, Mante FK, Chiche G, Saleh N, Takeichi T, Blatz MB. A retrospective survey on long-term survival of posterior zirconia and porcelain-fused-to-metal crowns in private practice. Quintessence Int 2014;45(1):31-38 